\section{Arquitectura de tierra, piedra y madera en Madrid (ss. V-IX d.C.). Variables materiales, consideraciones sociales}

\author{
Alfonso Vigil-Escalera Guirado
}

\begin{abstract}
Resumen
El trabajo pasa revista a los datos provistos por recientes excavaciones, en su mayoría inéditas, concernientes a tipos de arquitectura doméstica altomedieval documentadas en la región de Madrid. El empleo de materiales de construcción disponibles en el entorno inmediato de los asentamientos marca importantes diferencias entre territorios a veces muy próximos. Pero incluso en contextos como éstos, caracterizados por una significativa autosuficiencia y el «recurso a ciclos productivos simplificados» (AzKARATE, QUIRÓs, 2001: 53), se advierte un acceso y utilización diferencial de materiales que podría constituir un cierto indicador de formas de desigualdad en el seno de las comunidades.
\end{abstract}

Palabras Clave: Arquitectura doméstica, Poblados rurales, Época altomedieval, Materiales de construcción, Desigualdad social.

\footnotetext{
Abstract

This work reviews the data provided by recent excavations, mostly unpublished, concerning domestic architectural types of early medieval period from the region of Madrid. The use of raw material available in the proximity of the settlement marks major differences between very near territories.

Even in this kind of context, characterized by a significant self-sufficiency and the «employ of simplified productive cycles» (AZKARATE, QUIRÓS, 2001: 53), the differential access and use of raw material could provide us an indicator about certain forms of inequality in the community.
}

Keywords: Domestic architecture, Rural settlements, Early Medieval period, Contruction materials, Social inequality.
El panorama que ofrecen las excavaciones llevadas a cabo en el territorio de la Comunidad de Madrid durante estos últimos años, sobre todo por lo que respecta al poblamiento rural de época altomedieval, abre nuevas perspectivas en relación a la posibilidad de un estudio global de las comunidades campesinas: a la organización de sus procesos productivos, a su estructuración interna (espacial o social) e incluso a los aspectos ideológicos o políticos que subyacen en su desarrollo histórico. A un ritmo acelerado se están identificando nuevos yacimientos con evidencias de fórmulas arquitectónicas basadas en el empleo mayoritario de materiales perecederos o que recurren de forma casi exclusiva a los disponibles en el entorno inmediato del asentamiento. No cabe duda de que este fenómeno se relaciona con soluciones autoconstructivas por parte de las entidades familiares.

Como en todo campo nuevo de análisis, el ensayo de interpretación de los primeros casos documentados de arquitectura doméstica rural de época visigoda en el Sur de Madrid (VigIL-ESCALERA, 2000) chocó con el sesgo derivado del reducido tamaño muestral de lo que entonces podían parecer casos aislados y fuera de la norma. En la actualidad, y tras los primeros trabajos de síntesis y replanteamiento (AZKARATE, QUIRÓs, 2001), nos encontramos en mejor disposición para saber hasta dónde llega nuestro (des)conocimiento del fenómeno y sus implicaciones.

Frente a los esfuerzos clasificatorios exhaustivos del repertorio casuístico a escala continental (VALENTI, FrONZA, 1997), en estas líneas propondremos como alternativa más modesta de aproximación el intento de delimitación de algunas variantes del registro a escala regional o comarcal. Esbozaremos además unas reflexiones sobre cómo las variables observadas en cuanto al empleo y selección de materiales puedan constituir indicadores de desigualdad en contextos sociales aparentemente bastante homogéneos.

\section{CONSTRUCCIONES «DE SUPERFICIE» Y ESTRUCTURAS EXCAVADAS}

De acuerdo al cada vez mayor número de yacimientos excavados y de los datos disponibles, parece pertinente reconocer la posible existencia de una cierta dualidad (o diferencia conceptual original) entre las construcciones de uso residencial o auxiliar (no específicamente de almacenamiento) levantadas a nivel del suelo o "de superficie» y las que cuentan con suelos rehundidos intencionalmente o fondos excavados hasta una notable profundidad (Peytremann, 1995) ${ }^{1}$. A

\footnotetext{
${ }^{1}$ En este punto convendría no confundir el rehundimiento de los niveles de suelo del interior de espacios habitacionales por causas derivadas del mantenimiento y limpieza del mismo con los suelos rehundidos de estructuras construidas desde su origen con ese rasgo de forma intencionada.
} 
medida que aumenta el repertorio tipológico se advierte cada vez una mayor frecuencia, sin embargo, de tipos «mixtos» en el seno de esta última categoría, con lo que, a la postre, el criterio delimitador básico sería el establecido entre las edificaciones sobre zócalo pétreo provistas mayoritariamente de cubiertas de teja y el resto, diseñadas en función de cubiertas más ligeras, en materiales perecederos.

\section{Construcciones "de superficie»}

Los asentamientos altomedievales excavados (ss. V-IX d.C.) que disponen de registro arqueológico con construcciones de superficie en la región de Madrid (Gózquez, La Huelga, El Pelícano, La Vega o los poblados serranos ${ }^{2}$ ) nos muestran edificaciones provistas de zócalos de piedra sin concertar ni apenas desbastar, alzados preferentemente en tapial y cubiertas de teja curva. Los zócalos casi no presentan zanja de cimentación, o lo hacen de forma muy somera, y no se advierte el uso de morteros ni la existencia de pavimentos que no sean de tierra apisonada o el propio firme geológico regularizado. La existencia de este tipo de edificaciones se sospecha en otros tantos yacimientos, aunque no hayan podido ser documentados por causas relacionadas con la erosión superficial del terreno y pérdida de estratificación original ${ }^{3}$.

La documentación disponible acerca de edificios con zócalos de piedra muestra la posible coexistencia de dos modelos principales que se repiten de forma regular en diferentes yacimientos: la unidad de edificación de planta rectangular (EPR), a veces con una división interna en dos ambientes (en bastantes casos aparecen unidades yuxtapuestas), y la de planta compleja (EPC, con tres o cuatro ambientes diferenciados), en la que el rasgo más llamativo es la presencia de una habitación rectangular alargada y estrecha cerrando uno de los lados y una posible especialización funcional de los diversos ambientes. En la figura 1

\footnotetext{
${ }^{2}$ La mayor parte inéditos, sólo cuentan con la documentación aportada en informes preliminares de excavación o publicaciones parciales. El resto se recoge en la bibliografía final.

${ }^{3}$ Es el caso de Fuente de la Mora (Leganés), entre otros. Los materiales recuperados en el interior de silos o cabañas excavadas demuestran la demolición previa de edificios con zócalos pétreos y cubiertas de teja. Detrás de la asimetría numérica entre ambas clases de construcción (de superficie vs. de fondo rehundido) se esconden procesos tafonómicos, de conservación diferencial del registro, pero también metodológicos, dado que las condiciones del desbroce superficial mecánico en muchas intervenciones arqueológicas y las limitaciones estructurales propias de la arqueología de salvamento (cuestiones presupuestarias y de plazos de entrega de los trabajos) no propician precisamente el necesario respeto a la eventual presencia de estratificación horizontal, siempre de carácter precario, asociada a los asentamientos rurales.
}

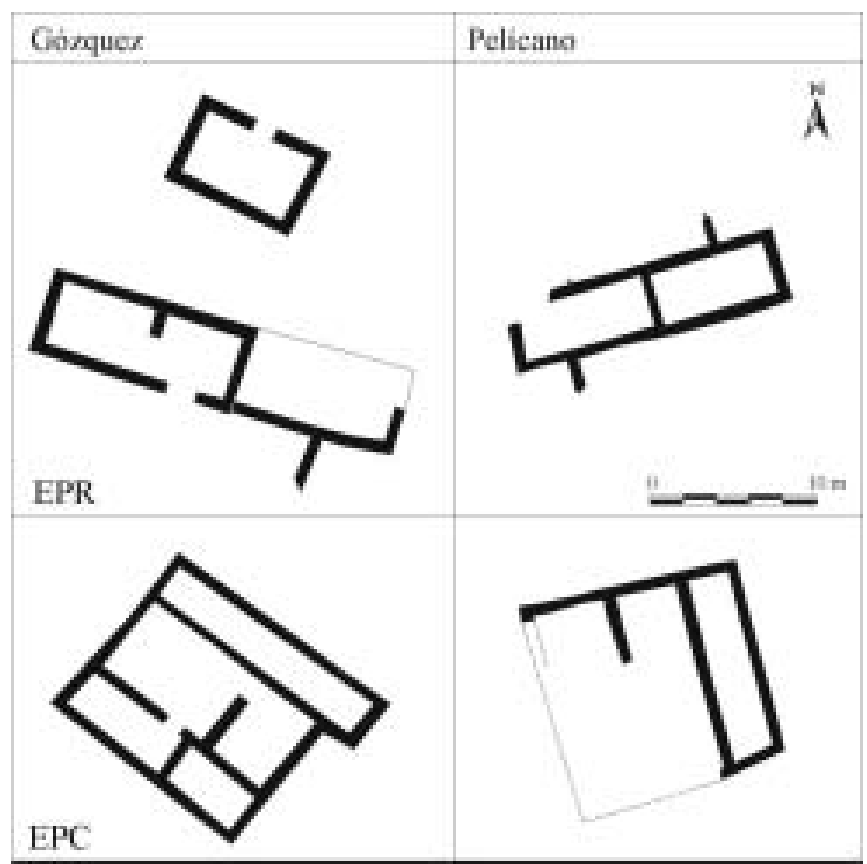

Fig. 1. Edificios de planta rectangular sencilla (EPR) y de planta compleja (EPC) documentados en dos yacimientos madrileños

se advierte la notable similitud entre plantas de construcciones de ambos tipos documentadas en los poblados rurales madrileños de Gózquez y El Pelícano. Sería reiterativo enumerar los yacimientos en los que se documentan edificios del tipo sencillo (EPR) y su amplia dispersión geográfica. En Madrid destacaremos los ejemplos de Navalvillar, La Vega, Gózquez y El Pelícano, y en el resto de la península los casos de El Tolmo (GuTiérREZ, 1999: 78 y Fig. 5) o El Cañal (STORCH, 1997). Asociados a ambos se registran de forma esporádica espacios cercados en torno a la unidad residencial.

\section{Construcciones de suelo rehundido o fondo excavado}

La adaptación a las condiciones regionales de clima continental bastante extremo (autorregulación térmica) y la sencilla excavabilidad del subsuelo determinan la frecuencia con la que se documentan estructuras de este tipo en amplias zonas del territorio madrileño. La mayor o menor disponibilidad de materiales pétreos conduce igualmente al empleo de los alzados de tierra (tapial) para las casas y del entramado vegetal con revoco de barro en las cabañas de las comarcas sedimentarias, mientras que se observa una utilización preferente y casi exclusiva de la mampostería (coincidente con la ausencia de estructuras de suelo excavado) en las zonas serranas. A escala regional, el panorama más reciente ofrece datos cada vez más abundantes de poblados con estructuras de suelo rehundido en los terrenos de la 


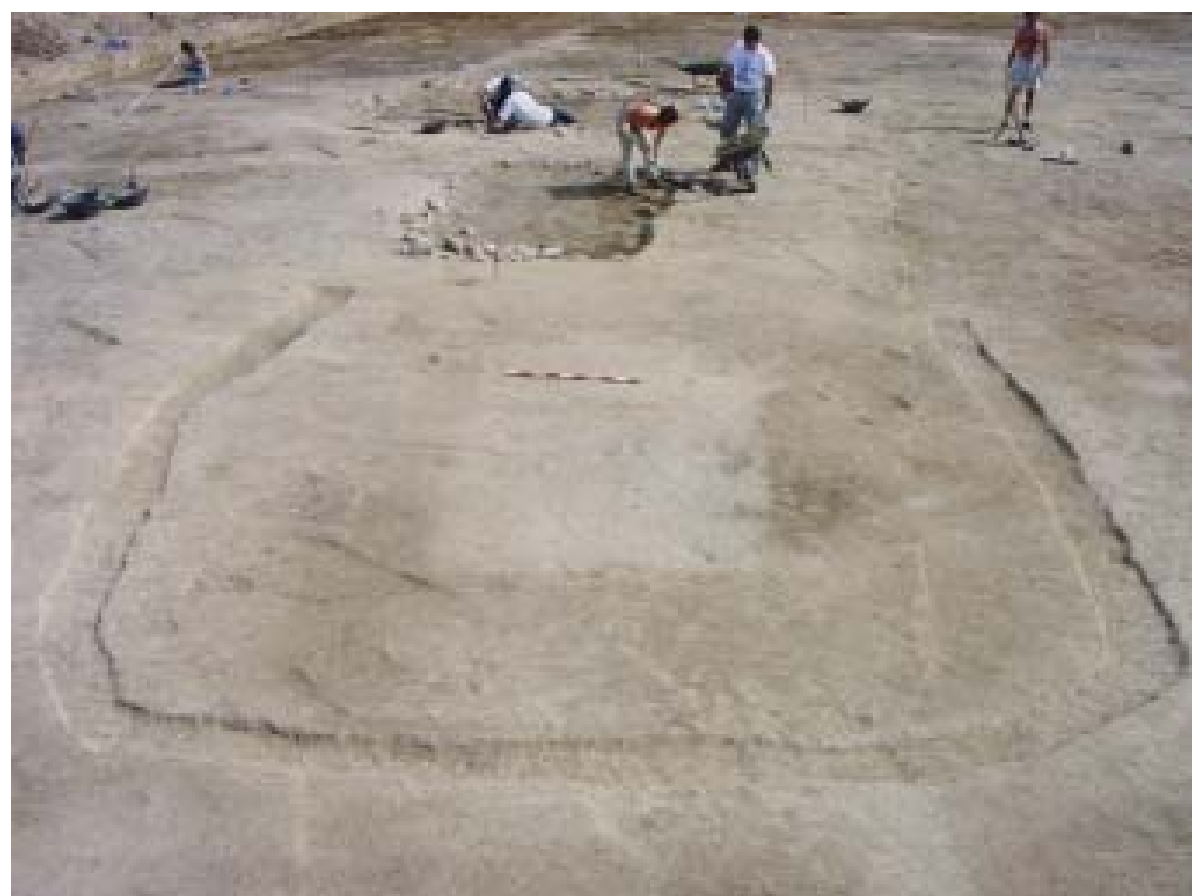

Fig. 2. Cabaña compleja de formato subrectangular, provista de roza perimetral exterior (Pelícano, sector 1), datada en el siglo vi d.C. El ambiente Norte, en primer plano, dispone de una estructura de suelo rehundido y planta rectangular en su interior cuenca sedimentaria o en las vegas de los ríos. La zona serrana, en la que abunda la piedra, resulta mucho peor conocida, aunque parece lógico que se reduzca el número de casos de estructuras excavadas (o simplemente desaparezcan) a expensas de una utilización masiva de la piedra como material constructivo.

Las escasas evidencias acerca de los materiales empleados en la construcción de alzados y cubiertas suelen derivar del hallazgo de estratos de aspecto «de piel de leopardo» sobre los niveles de suelo o abandono, estratos formados seguramente a partir de la descomposición de entramados vegetales con manteados de arcilla. El uso de tablas o madera se deja entrever a partir de las formas de algunas fosas de esquinas en ángulos vivos en las que además es más abundante la identificación de huellas de postes en los extremos axiales.

Las formas mixtas, en las que se recurre al empleo de zócalos o muros de mampostería desde el interior de estructuras excavadas, cada vez se documentan con mayor frecuencia. Normalmente aparece sólo una de las paredes de la fosa forrada con piedras, y en algún caso puede pensarse en la posibilidad de que se trate del zócalo de un muro sustentante al que se asociarían postes en otras partes de la cabaña.

En algunas intervenciones muy recientes (El Pelícano, en Arroyomolinos, con trabajos de campo en curso a junio de 2003) ha sido posible, además, documentar cabañas construidas con zanjas perimetrales que engloban a estructuras más simples. Las características de los casos documentados, asociados a fondos rehundidos en su interior e incluso a formas mixtas (fosas con una pared forrada de piedra), invitan a pensar que este tipo de construcciones fueron más frecuentes de lo que su aparición en el registro tiende a indicar. De nuevo son procesos tafonómicos y metodológicos los que condicionan la representación de tipos en un catálogo.

En el caso ilustrado, la planta completa de una cabaña queda dividida en dos ambientes: uno con roza o zanjita perimetral y huella de estructura de fondo rehundido rectangular en su interior, el otro de formato casi cuadrangular, bastante rehundido y con muro o zócalo de piedra en la pared medianera, al interior de la fosa. La disposición general del conjunto resulta muy similar a la de uno de los ejemplos vitorianos (AZKARATE, Quirós, 2001: Fig. 4.2), aunque en este caso, el perímetro queda marcado por agujeros de poste. (Figura 2)

\section{VARIABLES MATERIALES, CONSIDERACIONES SOCIALES}

El recurso preferente y casi exclusivo a los materiales disponibles en el entorno inmediato parece ir aparejado a soluciones autoconstructivas (sin que medien artesanos especializados) a lo largo de todo este periodo, al menos en ámbito rural. Y es en este contexto en el que el "valor» de los materiales puede configurarse como factor determinante en la ubicación de un determinado yacimiento a la vez que testimonio acerca de las diferencias económicas o de recursos de un individuo o familia dentro de una comunidad. Y para comprender mejor a lo que nos referimos por el «valor» de los materiales, nada mejor que exponer un par de casos. 


\section{PLANTA Y SECCRON FSQUEMATDCA DE LACABASA UE 1230}

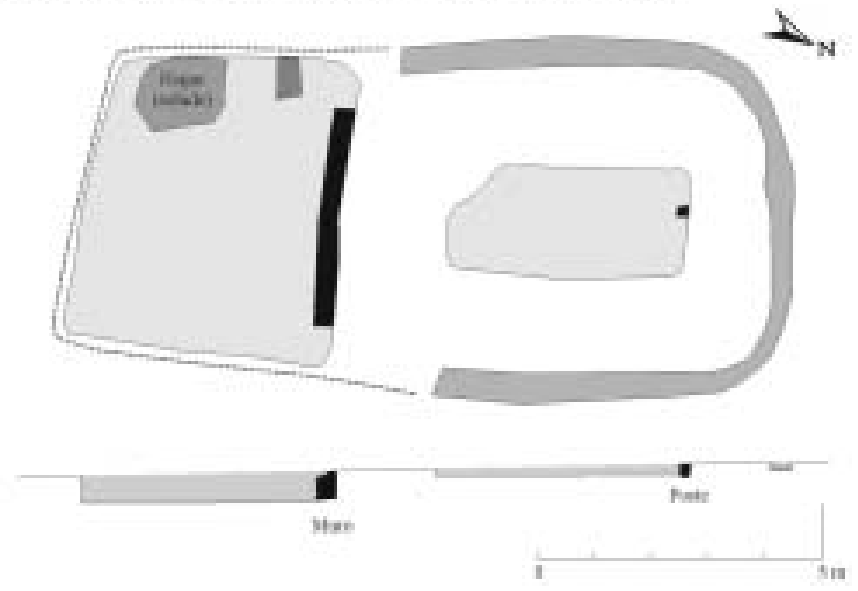

Fig. 3. Esta cabaña ejemplifica la complejidad que comienza a intuirse en lo relativo a los tipos constructivos de la arquitectura doméstica altomedieval. El estado de conservación del yacimiento influye de forma decisiva en el reconocimiento de nuevas morfologías

En La Indiana (Pinto) pudo comprobarse el saqueo altomedieval de una construcción de época altoimperial romana por medio de trincheras de expoliación y el uso sistemático de sus ruinas como cantera de piedra y quizás teja. En este caso resulta claro que no se pudo dar una reutilización de espacios abandonados (puesto que hay un abandono persistente y continuo del lugar durante casi tres siglos entre ambas ocupaciones), fenómeno que puede no verse con tanta nitidez en muchos otros yacimientos con fases tardorromanas. Resultaría interesante comprobar hasta qué punto el fenómeno no pocas veces descrito de reocupación de una villa romana por un cementerio o poblado de época visigoda no esconde en realidad la elección del lugar en función de la disponibilidad de materiales de construcción reaprovechables por el nuevo asentamiento.

En el yacimiento de Gózquez (San Martín de la Vega), todos los edificios con zócalo de piedra documentados pertenecen a las últimas fases de ocupación del asentamiento, pero también es cierto que existen edificios «en sombra», señalados por sutiles trincheras de planta ortogonal que delimitan restos de estratificación horizontal y podrían estar testimoniando una reutilización sistemática y exhaustiva de materiales pétreos y cerámicos procedentes de construcciones amortizadas, de modo que cada nueva edificación borra casi completamente las huellas de las preexistentes.

En el análisis de cada yacimiento singular, es precisamente la cuestión relativa a la efectiva variabilidad documentada en los materiales empleados en la construcción y su capacidad de ofrecernos un diagnóstico acerca de la capacidad económica diferencial del grupo o familia involu- crado en esa determinada construcción el ámbito en el que deben resolverse gran parte de las contradicciones y complejidades observadas, más allá de intentos de clasificación que, a la postre, pueden no tener más que un valor puramente coyuntural o casuístico.

De acuerdo a la entrevista ordenación familiar de la ocupación en los poblados, la delimitación de desigualdades en el seno de las comunidades rurales debe dirigirse hacia un doble objetivo: la de las diferencias existentes entre los miembros de una determinada agrupación familiar (por razones de edad, género o incluso condición social) y la que podría haber existido entre grupos o familias y comunidades vecinas.

Cuando a partir del siglo VII d.C. casi desaparecen los bienes personales del interior de las sepulturas, el modo en que se construyen éstas o los materiales empleados permiten intuir un acceso diferencial a recursos cuya explicación o interpretación no debería sustraerse al análisis de las desigualdades sociales.

Dirijamos ahora, pues, el discurso en torno a dos universos paralelos y cada vez mejor definidos a nivel arqueológico en el panorama madrileño: los cementerios de comunidades y los de tipo familiar. El análisis de éstos últimos permitirá adentrarnos en las desigualdades existentes en el seno de las entidades familiares; el estudio de los grandes cementerios de poblados podría dar pautas al respecto de las desigualdades entre familias a la escala del asentamiento.

En la vega del Jarama se han documentado asentamientos de carácter unifamiliar (La Huelga, en Barajas-Madrid, o Quintano, en Mejorada) y otros pertenecientes a comunidades plurifamiliares. Con cronologías que podrían abarcar desde mediados del siglo VIII d.C. hasta el siglo X, los cementerios familiares demuestran una amplia variabilidad de tipos constructivos y de materiales. El caso de La Huelga sería en ese punto sintomático: de las ocho sepulturas documentadas, prácticamente coetáneas según el estudio preliminar (VIGIL-EsCALERA, e.p.), cuatro pertenecen a individuos infantiles, las otras a jóvenes o adultos. Pues bien, una de las infantiles dispone de cubierta de tejas completas seleccionadas al efecto (incluso por su color) y una de las de adulto tiene la cubierta construida con grandes lajas de piedra. Ambos son recursos caros y escasos, y la piedra ha debido traerse desde una distancia de varios kilómetros. El resto de las tumbas presentan cubiertas de tabla de madera u otro producto perecedero. Considerando su práctica coetaneidad, las particularidades observadas (si optásemos por no atribuirlas al simple azar de los acontecimientos) habrían de responder necesariamente a variaciones en la consideración personal de los difuntos en el seno de la familia. 


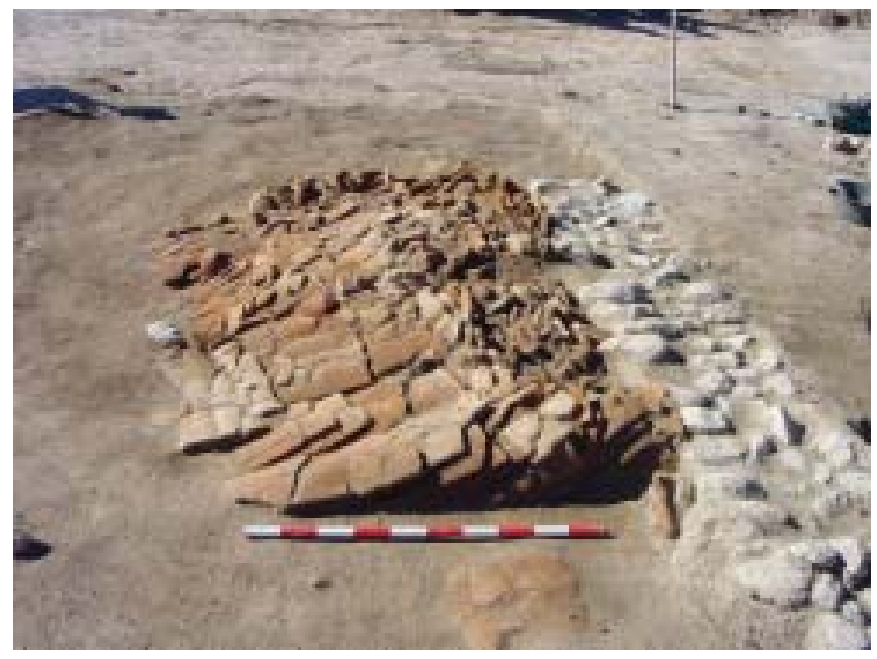

Fig. 4. Apilamiento de tejas listas para su reaprovechamiento. Proceden de un contexto altoimperial romano de abandono (Pelícano, sector 1) datado en la segunda mitad del siglo I d.C

Por la otra vertiente, la de los grandes cementerios de poblados con ocupación plurisecular, las fases avanzadas (del siglo VII d.C. en adelante) presentan no ya sólo graves problemas de reconocimiento y datación (CONTRERAS,VIGILEsCalera, e.p.) sino de indeterminación en general. Pero la ausencia de bienes personales que acompañen al difunto puede suplirse parcialmente a partir de la efectiva variabilidad de tipos constructivos y por los materiales utilizados en la construcción (la inversión efectiva amortizada en la parte del sepelio que pervive en el registro arqueológico). De este modo vemos cómo en las sepulturas más humildes, las fosas simples se cubren con tabla de madera mientras que las más costosas hacen una utilización profusa de grandes lajas de piedra en paredes, solados y cubierta, tratando de emular en lo posible los sarcófagos monolíticos de los estamentos más poderosos de la sociedad y la época.

El análisis de las desigualdades sociales a partir del registro arqueológico proporcionado por estos cementerios pasará, no obstante, por la discriminación en términos espaciales de las agrupaciones familiares o estamentales establecidas en el interior de los recintos de enterramiento, asunto de enorme complejidad y al que tal vez sólo la determinación del parentesco a través del análisis del ADN pueda aportar datos de calidad en el futuro.

Ante la falta casi absoluta de elementos de ostentación o "prestigio" asociados a la mayor parte de los enterramientos, el valor de los materiales utilizados en la construcción de las tumbas podría constituir una prueba circunstancial acerca de la capacidad económica de la familia del difunto o de su consideración social en el seno de la comunidad. Otro tanto podría decirse del análisis en clave social de los asentamientos (de cuya primera revisión sorprende siempre una

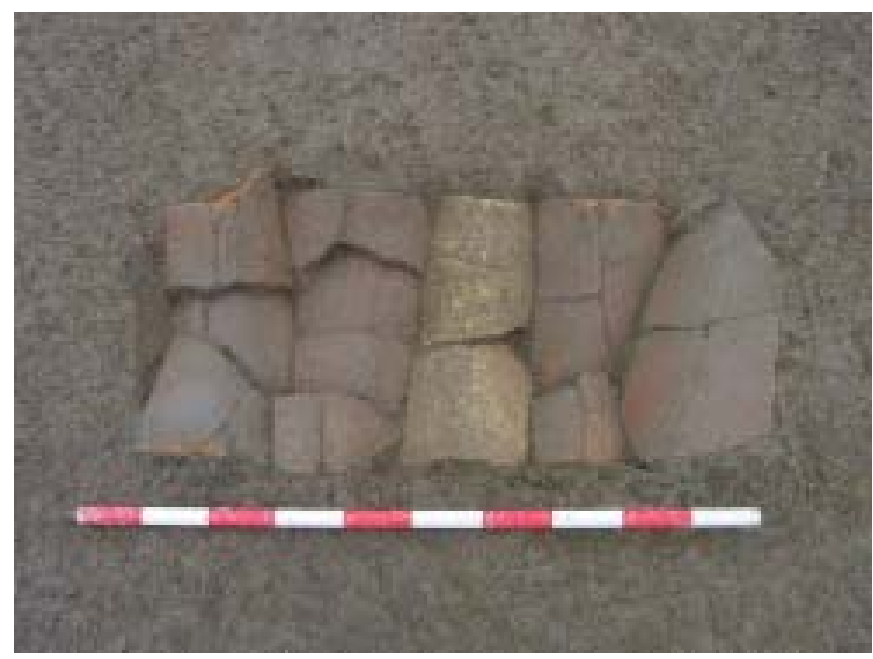

Fig. 5. Cubierta de tejas enteras perteneciente a una tumba infantil de rito islámico en la vega del río Jarama (La Huelga, Barajas, Madrid)

llamativa homogeneidad a escala interfamiliar) y en el que habrán de emplearse esfuerzos suplementarios a nivel metodológico e interpretativo si pretendemos optar a un conocimiento que supere el discurso meramente descriptivo.

\section{Bibliografía}

Alfaro M., BAÑón A., 2000, La Vega, asentamiento visigodo en Boadilla del Monte (Madrid), en RuANO, E. (dir.) La Arqueología madrileña en el final del siglo XX: desde la prehistoria hasta el año 2000, Boletín de la Asociación Española de Amigos de la Arqueología, 39-40, pp. 225238.

AZKARATE. A., QUIRÓs J. A., 2001, Arquitectura doméstica altomedieval en la península ibérica, Archeologia Medievale, XXVIII, pp. 25-60.

Caballero L., 1989, Cerámicas de «época visigoda y postvisigoda» de las provincias de Cáceres, Madrid y Segovia, Boletín de Arqueología Medieval, 3, pp. 75-108.

Contreras M., Vigil-Escalera A., e.p., El poblado visigodo de Gózquez (San Martín de la Vega, Madrid), IV Encuentro Internacional: Hispania en la Antigüedad tardía (Alcalá de Henares, 1999).

GuTiÉRrez Lloret S., 1999, La cerámica emiral de Madrinat Iyih (el Tolmo de Minateda, Hellín, Albacete). Una primera aproximación, Arqueología y Territorio Medieval, 6, pp. 71-112.

Peytremann E., 1995, Les structures d'habitat rural du haut Moyen Âge en France ( $\mathrm{V}^{\mathrm{e}}-\mathrm{X}^{\mathrm{e}} \mathrm{s}$.), en LORREN Cl. Y PERIN P. (eds.), L'habitat rural du haut Moyen Âge, Rouen, pp. 1-28.

STORCH J.J., 1997, Avance de las primeras actividades arqueológicas en los hispano-visigodos de La Dehesa del Cañal (Pelayos, Salamanca), Arqueología, Paleontología y Etnografía, 4, pp. 141-160.

VALENTI M., FronZa V., 1997, Lo scavo di strutture in materiale deperibile. Griglie di riferimento per l'interpretazione di buche e di edifici, I Congresso Nazionale di Archeologia Medievale (Pisa, 1997), Florencia, pp. 172-177.

Vigil-EsCALERA A., 2000, Cabañas de época visigoda: evidencias arqueológicas del Sur de Madrid. Tipología, elementos de datación y discusión, Archivo Español de Arqueología, vol. 73, pp. 223-252.

Vigil-EsCALERA A., e.p., Noticia preliminar acerca del hallazgo de una necrópolis altomedieval de rito islámico en la Comunidad de Madrid. El yacimiento de La Huelga (Barajas, Madrid), XXVII Congreso Nacional de Arqueología (Huesca, mayo, 2003). 